\title{
LEOPOLDO EIJO Y EL INSTITUTO FRANCISCO SUÁREZ DEL CSIC
}

\author{
POR \\ SANTIAGO MATA \\ Dowina, Bratislava
}

\section{Resumen}

Estudio del impulso que el obispo de Madrid, Leopoldo Eijo, dio a la creación (1940) y a las actividades del Instituto de Teología "Francisco Suárez", del Consejo Superior de Investigaciones Cientificas, en Madrid, hasta su muerte en 1963. Destaca su sensibilidad ante la posible penetración de la nouvelle théologie en España.

\section{Abstract}

Leopoldo Eijo and the "Instituto Francisco Suárez" from the CSIC

Study about Madrid bishop Leopoldo Eijo's effort for creation (1940) and works of "Francisco Suárez" Institute of Theology, CSIC, in Madrid, until his death in 1963. It is specially important his caution in face to the penetration of nouvelle théologie in Spain.

Nacido en Vigo en 1878, Leopoldo Eijo Garay era obispo de Madrid desde 1923, y en 1940 se hizo cargo de la dirección del recién fundado Instituto de Teología del Consejo Superior de Investigaciones Científicas. Ingresado tempranamente en el Seminario de Sevilla, el joven gallego había vivido en el Colegio Español de Roma desde 1892, es decir, al año siguiente de su crea- 
ción. En las universidades romanas destac6 particularmente por sus dotes intelectuales'.

De regreso a España, a los 27 años de edad, Eijo fue nombrado capellán de Alfonso XIII, quien lo eligí como obispo de Túy en 1914, y de Vitoria tres ańos más tarde. Antonio Maura manifestó en 1920 su deseo de que Eijo fuera elegido miembro de la Academia de la Lengua, deseo que no se hizo realidad hasta 1927. Durante la Dictadura de Primo de Rivera, Eijo intervino en el ámbito de la enseñanza universitaria formando parte del Consejo de Instrucción Pública. Ya en el período republicano -en 1935-, el obispo de MadridAlcalá ingresó en la Academia de Ciencias Morales y Políticas².

\section{EUO Y LA INVESTIGACIÓN CIENTÍFICA}

Una simple enumeración de los cargos que Eijo ocupó sería insuficiente para hacernos cargo de sus ideas acerca de la investigación científica. Las obras de carácter científico del que durante más de cuarenta años había de ser obispo de Madrid-Alcalá son todas de tipo teológico, y particularmente de historia, con un interés muy especial por la figura de Ramón Llull. Así pues, Eijo no expresó un pensamiento concreto sobre la misión que, a partir de 1940 , podía competir al CSIC, y dentro de él a las ciencias humanas. Pero sí resaltaba - por ejemplo en una

I La documentación de este artículo puede verse en la tesis doctoral que, con el título Leopoldo Eijo Garay (1878-1963) defendí en la facultad de Filosofía y Letras (sección de Historia) de la Universidad de Navarra el 14 de diciembre de 1995. Agradezco en particular a D. Horacio Santiago ( $\dagger$ ) las facilidades que tuve para consultar el Archivo del Instituto Francisco Suárez (AIFS).

2 Maura expresó este deseo después de escuchar la Oración fúnebre pronunciada por el Iltmo. Sr. Dr. D. Leopoldo Eijo Garay Obispo de Vitoria en la iglesia de las Religiosas Trinitarias de Madrid el dia 23 de abril de 1920 en las solemnes exequias que por el alma de Miguel de Cervantes Saavedra y de cuantos cultivaron gloriosamente las letras patrias celebro la Real Academia Española. La principal obra de investigación que para entonces había publicado Eijo era El primer origen de la vida según el Hexámeron y la Ciencia (Santiago de Compostela, 1913, Seminario, 90 páginas).

Antes de su discurso de ingreso en la Real Academia (Madrid, 1927, talleres "voluntad", 58 páginas), Eijo -que desde 1905, ocupando la vacante de José María Ortí y Lara, era miembro de la Academia Romana de Santo Tomás de Aquino- pronunciaría también una conferencia sobre Santo Tomás y la Mística (20 de noviembre de 1923) en la Semana Tomista de Roma. Antes de su discurso de ingreso en la Academia de Ciencias Morales y Políticas publicaría un Esbozo apologético de la poesia eucarística clásica española (páginas 369 a 394 de Revista Eclesiástica - Órgano del clero de habla española-, $3^{2}$ época, Año IV, vol. IV, Madrid, 1932, Talleres Luz y Vida), y un amplio prólogo (páginas I-XLIX) al libro de Francisco SUREDA BLANES, El beato Rambn Llull (Raimundo Lulio). Su época. Su vida. Sus obras. Sus empresas. Madrid, 1934, Espasa Calpe, 392 páginas, Biblioteca Nacional, 1/203771.

Cincuenta af̃os

Hispania Sacra 49 (1997) 
conferencia dictada en 1923 - la importancia del correcto raciocinio en los estudios teologicos:

\begin{abstract}
"Porque, dada la naturaleza humana, aun las mismas doctrinas sobrenaturalmente reveladas, requieren nuestra razón para conservarse puras entre los hombres, puesto que al discurrir sobre ellas, al estudiarlas y sistematizarlas, al traducirlas en lenguaje de enseñanza o de defensa, al asimilarlas a nuestra vida para que sean, como deben ser, principio y norna, sostén y aliento de toda ella, tienen que vaciarse y traducirse en el molde de nuestros conocimientos naturales; de modo que, si hay en éstos error, se convierte en error teologico el que era puramente filosófico"3.
\end{abstract}

Eijo, a semejanza del personaje medieval a quien más estudios dedicara - Ramón Llull- tenía en mucho respeto a la razón y a la ciencia, por considerar que ayudaban a conocer y difundir la fe. Terminada la contienda civil española, el obispo de Madrid trató de poner en práctica una aspiración que había expresado en tiempos de la República: la de que Madrid contara con una institución teológica de rango universitario: un "Centro de Estudios Religiosos, digno de la capital de España"4.

Conviene distinguir la institución en cuya creación y desarrollo participaría Eijo -el Instituto Francisco Suárez del CSIC — de las que se dedicarían a la formación religiosa de los laicos —que es el contexto de la frase citada-, y del seminario, aunque éste fuera también un centro de estudios eclesiásticos.

${ }^{3}$ L. EuJo, Santo Tomás y la Mistica, p. 32 y 12. Para Salvador Muñoz Iglesias, Eijo "era un enamorado, personalmente, de la filosofia aristotélica, y pensaba que lo mejor que se podría hacer por la cultura moderna era imbuirla de aristotelismo. Conversación el 22 de marzo de 1988. La auténtica devoción de Eijo por Llull (1233-1316) se explica precisamente por el deseo, común al obispo gallego y al beato mallorquín, de armonizar ciencia y fe (o viceversa). Los principales estudios (seis de ellos publicados póstumamente) de Eijo sobre Llull son:

"La finalidad de la Encarnación según el beato Raimundo Lulio". En Revista Española de Teolo" gia, II, 1942, p. 201-227.

"Las razones necesarias del Beato Ramón Llull en el marco de su Epoca”. Páginas 23-38 de Estudios Lulianos, 9 (1965).

"La supuesta heterodoxia del Beato Llull". Páginas 5-19 de Estudios Lulianos, 12 (1968).

"El Doctor Amor Ruibal y su juicio sobre el Beato Lulio". Páginas 227-235 de Estudios Lutianos, $14(1970)$.

"La luz divina en la gnoseología luliana". Páginas 153-173 de Estudios Lulianos, 15 (1971).

"Iluminismo ixraquí?", páginas 61-79 de Estudios Lulianos, 17 (1973), enero-mayo.

"Las dignidades lulianas". Páginas 26-46 de Estudios Lulianos, 18 (1974), enero-diciembre.

4 Eijo quería "acabar con la ignorancia religiosa de tantos católicos inconscientes que casi desconocen la fe que profesan, que asisten a cultos sagrados que apenas entienden, y que si bien, en el mejor de los casos, están prontos a dar la cara por su religión, no pueden dar razones que la ilustren y la vindiquen." Boletín Oficial del Obispado de Madrid-Alcalá (en adelante citado como BOOMA), 1933, p. 314 . 
Para enfrentarse a las carencias de la formación religiosa, y en concreto para formar maestros de escuela católicos, Eijo había fundado en 1926 la Institución del Divino Maestro. Más tarde, cuando la crisis se agudizara, fundaría los "Cruzados de la Enseñanza"5. Ambas instituciones tenían como objetivo la educación religiosa elemental.

Por lo que se refiere al seminario, la diócesis de Madrid acusaba una grave escasez de sacerdotes, agudizada por el asesinato de 435 de ellos $-39 \%$ del total- durante la guerra. Ello hizo que, después de la contienda, no pudiera imponerse - suponiendo que se pretendiera- en el seminario de Madrid un exigente criterio de selección intelectual. La prioridad era "cubrir huecos", y los urgentes problemas pastorales a los que se enfrentaba la diócesis hacían pensar que, en caso de buscar para Madrid un centro de investigación teológica de rango universitario, no podría contarse para ello con el seminario. ${ }^{6}$

\section{"MAESTROS DEL MUNDO"}

La solución se la brindaría al obispo de Madrid su buen amigo y ministro de Educación Nacional, José Ibáñez Martín, al fundar el Consejo Superior de Investigaciones Científicas y, dentro de él, el Instituto Francisco Suárez de Teología, que Eijo iba a presidir. A juzgar por las palabras que pronunció lbáñez Martín en la primera reunión plenaria del CSIC, el proyecto de Eijo gozaba de una posición de auténtico predominio:

\footnotetext{
"Subrayemos la necesidad y oportunidad de este Instituto [Francisco Suárez] para resucitar el empuje imperial de aquella teología que presidió todo nuestro saber en siglos dorados y que, resonando por boca de nuestros sabios en las cátedras universitarias de Europa y aun del Nuevo Mundo, fue el instrumento más poderoso de la expansión de la cultura hispánica. El Nuevo Estado cumple en eso un deber de conciencia nacional.
}

5 Sobre la creación de los "Cruzados de la Enseñanza" puede leerse BoOMA, 1933, p. 285, también BOOMA, 1956, p. 486.

6 Sobre los sacerdotes asesinados durante la guerra, cfr. José Luis AlfayA, La díocesis de Madrid-Alcala durante la guerra civil: 1936-1939. Pamplona, 1987 (tesis doctoral defendida el 1 de junio de 1987 en la Facultad de Teología de la Universidad de Navarra), 794 páginas, p. 500 y $709-748$.

El seminario de Madrid no destaca entre los españoles de posguerra en cuanto al aumento de sus alumnos, aunque éstos se duplicarían entre 1934 y 1947. Una de las causas sería la falta de recursos para atender a las vocaciones. Cfr. Severino AZNAR, La Revolución española y las vocaciones eclesiásticas, Madrid, 1949, Instituto de Estudios Políticos, 276 páginas, p. 83-102 y p. 69. Se comprende que Federico Sopeña hablara "de un seminario serrano, campesino, de un Seminario que no conto, que era como nada en la vida intelectual madrilefía, incluso en la época del triunfalismo". F. SOPENAA, Defensa de una generación, p. 35-36. Cfr. F. SOPEÑA, Escrito de noche, Madrid, 1985, Espasa-Calpe, 191 páginas, p. 107-109.

Cincuenta años

Hispania Sacra 49 (1997) 
Porque no sólo acata la jerarquía de la ciencia sagrada, otorgándole el puesto de honor que en el árbol de las ciencias le corresponde, e incorporando de modo pleno la tradición al actual renacimiento científico, sino que anhela, como deseaba Menéndez Pelayo, inyectar nueva savia a todas nuestras actividades culturales, para que la ciencia nacional sea así rotundamente católica y sirva ante todo los altos intereses espirituales de Dios y de su Iglesia."?

El entusiasmo de Eijo no le íba a la zaga al del ministro, y así se afirmaba desde las páginas del primer cuaderno de la Revista Española de Teología, publicado en octubre de 1940:

"No agradeceremos nunca harto tanta justicia y favor, si no ponemos rápida y esforzadamente manos a la obra de hacer resurgir los estudios eclesiásticos hasta la altura en que lucieron cuando los maestros españoles eran maestros del mundo."

A sus 62 años de edad, Eijo no veía en este proyecto una mera ocasión de figurar, pues en opinión de Joaquín Blázquez, que había de ser su secretario en el Instituto Francisco Suárez, "se entregó con toda el alma a todos y cada uno de los cargos" que ejerció en el CSIC:

"No era el Patriarca hombre al que le fuese lo de figura decorativa. Tenía una envidiable capacidad de trabajo y estaba dotado de poderosa, ágil y sutil inteligencia. Pasaba de un asunto a otro bien dispar sin el más mínimo esfuerzo. Además de que no descuidaba la conveniente preparación próxima y puesta a punto. Y sobre todo, no le apuraba nunca preguntar para informarse convenientemente y consultar otras opiniones para fijar o modificar la propia. Solía repetir con frecuencia que Dios premia con el acierto al que tiene la humildad de consultar."

\section{EL EQUIPO Y SUS PRIMEROS PROYECTOS}

La plantilla del Instituto Francisco Suárez (IFS) era de tres personas: Eijo, como director, Daniel García Hughes, canónigo de Madrid y profesor del se-

7 J. BLÁZQUEZ, "In memoriam. Excmo. y Rvdmo. Sr. Dr. D. Leopoldo Eijo Garay, Patriarca Obispo de Madrid-Alcalá, primer Director del Instituto", páginas 208-217 de Estudios Bíblicos, 1963, p. 214. Cfr. Memoria del Consejo, años 1940-1941, Madrid 1962, p. 47-48 para el discurso del ministro.

8 J. BLAZZUEZ, "In memoriam...", p. 213-214, cfr. Revista Española de.Teología, 1 (1940-41), p. $8-9$.

9 J. BLÁZQuEZ, "In memoriam....", p. 212. La lista de cargos de Eijo en el CSIC, ibidem, p. 211-212. En 1940, Eijo no era Patriarca de las Indias Occidentales, nombramiento que recibió en 1946, pero muchas fuentes escritas posteriores a 1946 lo designan así, incluso refiriéndose a periodos anteriores. 
minario, como vicedirector, y el mencionado sacerdote Joaquín Blázquez Hernández, coadjutor de Leganés, catedrático de teología en el seminario y secretario del IFS, quien anota que también colaboró desde los primeros momentos el futuro obispo de Mallorca, Jesús Enciso Viana. Un número reducido que pretendía ser el germen de algo mayor, más bien que un coto o una restricción impuesta por la escasez de medios. ${ }^{10}$

La primera actividad organizada por el IFS fue una Semana Bíblica - del 16 al 22 de septiembre de 1940, en Zaragoza, coincidiendo con el XIX centenario de la Virgen del Pilar-que debiera haberse celebrado en septiembre de 1936 en Salamanca. Con ella, Eijo quiso reorganizar la Asociación para el Fomento de los Estudios Bíblicos en España (AFEBE), lo que significaba coordinar con el IFS a esta asociación y a "sus dos empresas principales: la traducción íntegra de los textos originales al castellano y la revista Estudios Bíblicos, para que sirva de estímulo, de cauce a nuestros trabajos e investigaciones y para que levantada y sostenida por todos, sea el portavoz de la ciencia bíblica española, y adquiera gloria y prestigio para España en el campo internacional."1]

La Semana contó con las bendiciones de dos cardenales, tres arzobispos y tres obispos, y las inscripciones de 17 obispos o administradores apostólicos, 18 lectorales y profesores de Sagrada Escritura, y unos cincuenta religiosos, entre ellos 9 venidos del extranjero, aunque no extranjeros. ${ }^{12}$

A partir de octubre, lo que Eijo llamaba "Mesa del Instituto" cambió su sede provisional en el seminario por el palacio episcopal, donde se reunía todos los lunes de siete a diez de la tarde. La idea de celebrar, a semejanza de la Semana Bíblica, una Semana de Teología, surgió, "en una de las reuniones de la Mesa del Instituto en el mes de noviembre de 1940".13

Así pues, en su primer año de existencia, la modesta "Mesa" del IFS había dado a luz las que habían de ser sus dos principales iniciativas, las Semanas de Estudios Bíblicos y de Teología, que harán que, veinte años más tarde, José

10 J. BlázQuez, en la revista Espiga, 1958, p. 4. Blazquez había nacido el 14 de agosto de 1909 en Villa del Prado. Cfr. AIFS, expediente 2233. El IFS se constituyó por Orden Ministerial el 14 de marzo de 1940. Eijo tomó posesión a finales de junio. Su instalación provisional en el setninario no iría en contradicción con el afán selectivo, "pues anhelaba el Sr. Patriarca que los seminaristas se acercasen y que los mejor dotados se entrenasen en la investigación teológica y bíblica". Ibidem. De que la escasez era más de personal que de medios, da idea que Eijo contestara a la única carta de felicitación por el primer número de la Revista Espafiola de Teologfa que se conserva en el AIFS - escrita el 16 de enero de 1941 por eI P. Juan Prado, redentorista de Astorga, afirmando que el artículo publicado por Eijo "puede abrir nuevas rutas orientadoras a la historia de la Teologia"-, "pidiéndole nombres de colaboradores" (21 de enero de 1941). AIFS, expediente 45.

II BOOMA, 1940, p. 162. Cfr. AIFS, expediente 2.

12 BOOMA, 1940, P. 210. Cfr. J. BLÁZQUEZ, "In memoriam...", p. 215.

13 J. BlÁzQUEz, "In memoriam...", p. 214. Cfr. Espiga, 1958, p. 4.

Cincuenta affos

Hispania Sacra 49 (1997) 
María Casciaro califique al IFS como "el centro quizá más interesante", hasta el punto de suponer el comienzo de una nueva etapa en un campo, el de los estudios bíblicos y teológicos, que desde el impulso dado por León XIII sólo habría experimentado en España "una tímida tentativa de renacimiento":

"En los centros de estudios eclesiásticos de la península lbérica se enseñaba y se aprendía, en esta época, una teología sobre todo especulativa; se repetían y aprendian los tratados de los grandes maestros del pasado. Faltaban a la vez el impulso creador y el gusto por la investigación. En esta época, la teología española se limita a defender, en obras apologéticas generalmente de poco valor, la ciencia cristiana tradicional. Faltan a un tiempo la cultura y el impulso necesarios para plantear problemas nuevos y aportar nuevas soluciones. Esta situación dura aproximadamente hasta 1940."14

\section{UN ASUNTO ESPINOSO: LA CENTRALIZACIÓN}

La primera Semana Española de Teología, cuya organización se había acordado en noviembre de 1940 , se celebró en la segunda quincena de septiembre de 1941, coincidiendo con la segunda Semana Bíblica, que daba continuidad a la celebrada en Zaragoza. Según Joaquín Blázquez, constituyeron "el primer tacto de codos de los teólogos españoles después de nuestra Guerra. Fue como una especie de recuento de los supervivientes de las interminables jornadas gloriosamente trágicas y siembra para el futuro."15

La conyuntura bélica y la neutralidad oficial de España no eran óbice para que Eijo buscara el refrendo internacional de su iniciativa por parte de la autoridad que más le interesaba, y así las Semanas de Estudios Superiores Eclesiásticos de 1941 se abrían con un telegrama enviado por el Secretario de Estado de la Santa Sede al Nuncio en Madrid -13 de septiembre de 1941-,

14 J.M. CASCIARO, "Les études bibliques et théologiques dans l'Espagne actuelle", La Table Ronde, $n^{\circ} 144$, diciembre 1959, páginas 117-122, p. 117. Casciaro afirma que "el número de trabajos de investigación publicados entre 1939 y 1959 es dos veces mayor que el de los publicados entre 1900 y 1939, pero sobre todo es su calidad la que ha mejorado de forma imprevisible." Cita las dos revistas del Instituto en primer lugar entre las más relevantes y las semanas teologicas y bíblicas cómo únicos congresos o reuniones periódicas:

"Las Actas de estas semanas constituyen ya una importante colección de trabajos de investigación sobre los temas examinados. Pero quizá más aun que todos los trabajos realizados hasta ahora, esas semanas han sido precisamente una de las levaduras más activas para la vida intelectual de las Ciencias bíblicas y teológicas en España." lbid., p. 119.

Is J. BLÁZZUEZ, en Espiga, 1958, p. 4. Eijo había participado también en la preparación de una tercera semana, mariologica, a la que sin embargo no podría acudir el obispo, por celebrarse antes que las otras, y que no volvería a tener conexión con las semanas organizadas por el IFS. AIFS, expediente 42. 
cuyo tono no era menos eufórico que las declaraciones hasta ese momento hechas por los propios organizadores:

"Es digno de toda alabanza el propósito de dicho Instituto, órgano del Consejo Su" perior de Investigaciones Científicas para las cuestiones teológicas, de contribuir con estos actos al importante fin de renovar la gloriosa tradición científica sagrada... Ante este generoso esfuerzo por el resurgir de los estudios sagrados, el Augusto Pontífice, a la par que felicita a los organizadores de estos actos, formula sus más fervientes votos, no sólo por el éxito de las Semanas, sino también para que cooperando a este movimiento científico, en estrecha unión y en la medida de sus fuerzas, todos los miembros del Clero, particularmente el profesorado de los Seminarios, puesta la mira en los bienes inmensos que produce a la Religión y a las almas el cultivo de la ciencia sagrada, hagan volver pronto aquellos tiempos en los que España dio a la Iglesia Católica un número tan elevado de teólogos y exegetas insignes."16

El IFS contaba, pues, con todas las bendiciones -también en sentido literal- para organizar la investigación teológico-bíblica en España. Para comprender en qué consistiría esa "organización" - si era un mero título honorífico, o si se pensaba en un férreo control centralizado, si convendría mejor considerarla como coordinación, colaboración o dirección ejecutiva- sería preciso conocer las demás instituciones e iniciativas orientadas a la investigación existentes en ese momento, y lo que para ellas iba a suponer la creación del CSIC, del que formaba parte el IFS.

Por lo que respecta al CSIC, parece claro que, ya en este momento, su objetivo de centralizar la investigación suponía un recorte para las pretensiones de otra institución en cuyo origen Eijo había estado presente, y en cuyo desarrollo iba a estarlo aún más: se trata del Instituto de España. Formado en plena guerra civil por "las Comisiones de las Reales Academias de España", designadas por decreto el 18 de diciembre de 1937. Su acto de constitución tuvo lugar "en Burgos, en el local de su Instituto de Segunda Enseñanza, el día 27 de diciembre de 1937, a las 11 de la mañana, bajo la presidencia del Presidente de la Comisión de Cultura y Enseñanza, D. José María Pemán”. El 6 de enero de 1938, Eijo había actuado como testigo del juramento de los miembros de las Reales Academias que se adherían al régimen de Franco, en la primera sesión

16 En J. BLÁZQUEZ, "In memoriam...", p. 216. En la clausura de las Semanas, el nuncio felicitaría "de modo especialísimo al Excelentísimo Sr. Obispo đe Madrid, alma, guía, sostén y aliento de estas dos Semanas, para cuyo mayor esplendor ha puesto a contribución los tesoros de su vasta cultura y el fuego de su celo pastoral". Ibid., p. 216-217.

Cincuenta años

Hispania Sacra 49 (1997) 
solemne del Instituto de España, que presidió en el paraninfo de la Universidad de Salamanca el conde de Jordana ${ }^{17}$.

Como un reflejo - -entre otras cosas - de la salida de Pedro Sainz Rodríguez del gobierno, la creación del CSIC por su sucesor iba a poner en sordina la autoproclamada misión del Instituto de España de ejercer "en nombre del Estado, la tutela superior de las investigaciones científicas y de toda la enseñanza superior, en lo que no tenga carácter universitario." Si en 1938, Franco había sido representado por su ministro de Asuntos Exteriores en la inauguración del Instituto de España, el 17 de diciembre de 1941, asistiría en persona a la clausura de la segunda reunión del Pleno Anual del CSIC ${ }^{18}$.

Pues bien, podemos decir que Eijo iba a ser el encargado de garantizar que las aspiraciones del Instituto de España se mantenían dentro de sus nuevos límites, o si se prefiere una interpretación menos radical - y más conforme con el alma gallega de Eijo, que dominaba el arte de compatibilizar lo que parecía incompatible-, simplemente de armonizar las relaciones entre estas dos entidades.

En efecto, en este momento Eijo vuelve a asistir a una sesión solemne del Instituto de España (la decimocuarta) y toma parte en sus actividades, hasta hacerse cargo, el 25 de febrero de 1942, de la presidencia del Instituto, en un momento de total ruptura de los miembros de la mesa con el que era su "Secretario Perpetuo", Eugenio d'Ors. En esa primera reunión presidida por Eijo, se decidió suprimir el servicio de publicaciones del Instituto de España ${ }^{19}$.

Volviendo ya a referirnos al IFS, podemos comprender en qué consistian sus ideas sobre la coordinación investigadora, leyendo la correspondencia que mantuvieron en el verano de 1942 Eijo y Blázquez, para preparar la organiza-

17 La cita del acto de constitución, en el archivo del Instituto de España (AIdeE), librol, fol. 1R, cfr. 1V, 3V, 6R, 8R, 23R y 14R. Cfr. A. ALTED, Política del nuevo estado sobre el parrimonio cultrral y la educación durante la guerra civil española. Madrid, 1984, Ministerio de Cultura, 391 páginas; p. 239-40. Los estatutos del Instituto de Espafia no serían publicados hasta 1947 (BOE de 5 de mayo). E. VEGAS, Los caminos del desengafio. Memorias polfitcas 2. 1936-1938. Madrid, 1987, Giner, 530 páginas, p. 280-2, atribuye la idea del Instituto a Eugenio d'Ors y Sainz Rodríguez, aunque menciona también a Pernán. Sobre el juramento, cfr. AldeE, LI, fol. $34 \mathrm{~V}$ y fol. $4 \mathrm{~V}$.

18 La cita sobre la misión el Instituto de España, en AIdeE, librol, fol. 83V. Cfr. fol. 156R, $163 \mathrm{~V}, 165 \mathrm{R}, 55 \mathrm{~V}, 109 \mathrm{R}, 114 \mathrm{R}$ y $153 \mathrm{R}$. La supresión del servicio de publicaciones fue producto de las tensiones internas entre sus directivos, y no puede verse en elia un recorte de las actividades impuesto desde fuera. De hecho, en esa reunión Eijo anunció que trataría de conseguir una sede para el Instituto, y asî to hizo.

19 En la dacumentación del Instituto de España se pasa como sobre ascuas sobre la desaparición de Pedro Sáinz Rodríguez que, en rigor, nunca tuvo el cargo de presidente (pues to era Manuel de Falla, que desde un principio excusó aceptar, alegando enfermedad). Cfr. AldeE, L1, fol. 4R, 9R, 146R, 157R, 180V, 198V, AIdeE, L1A, p. 251 y 253. 
ción de las Semanas de Estudios Superiores Eclesiásticos de ese año. Eijo expresa su deseo de que el IFS coordine la labor de los mejores centros españoles de investigación teológica o bíblica y de que esté abierto al mayor número posible de colaboradores, dentro de su modesta capacidad económica:

"Dice V. bien que desde el principio sueño con un Instituto Suárez moldeado de esa forma. No puede el Instituto ser cosa de cuatro señores, y mucho menos si ya uno es tan inútil como yo, aunque pueda suplir la dignidad episcopal. Si en Madrid pudieran vivir todos esos Sres., y dedicarse a nuestros trabajos, sería cosa excelente; pero mejor es que vivan fuera dirigiendo diversos focos de investigación."20

La referencia de Eijo a esos "focos de investigación", y el mismo hecho de que el IFS se planteara la posible agregación de centros al IFS, no era un planteamiento teórico, ya que meses antes se les había propuesto la posibilidad de erigir una sección bíblica del Instituto en Zaragoza, conforme a un proyecto que venía con el aval del arzobispo de la capital aragonesa. Eijo y sus colaboradores consideraron que no era "necesaria, ni siquiera conveniente" esta sección, puesto que la ya existente en Madrid "debe seguir siendo única, [...] no sólo no excluye a nadie sino a todos ofrece facilidades y respetos":

\begin{abstract}
"Creemos que todos los frutos peculiares que se asignan en el referido Proyecto a la Sección Bíblica de Zaragoza, se pueden obtener, y seguramente mejor, colaborando cada uno en particular con la Sección Bíblica del Instituto; sin que esto quiera decir que no puedan prestarse mutua ayuda estando tan cerca unos de otros y tan unidos y acordes entre sí como se dice." 21
\end{abstract}

Así pues, por el momento, el IFS consideraba mejor la cooperación de las personas singulares que la de los "focos de investigación": no está claro si sus directivos pensaban que la propuesta de Zaragoza carecía de suficiente calidad -algo que parece sugerir el hecho de que no esperaran mayores frutos del trabajo colectivo que del individual-- o si, simplemente, la propuesta les cogio por sorpresa, en cuyo caso sería éste el motivo por el que inmediatamente se propusieron regular la agregación de nuevos centros. Puede pensarse, en contra de lo que afirman ellos mismos, que existiera un afán exclusivista. Pero existe al menos otro factor a tener en cuenta: la escasez de recursos económicos.

20 Carta de Eijo el 6 de septiembre, en AIFS, expediente 68. Estas ideas están presentes también en las correcciones que hace el obispo al proyecto de agregación de otros centros de investigación de ciencias eclesiásticas, redactado por Blazquez. Cfr. AIFS, expediente 75 bis y expediente 46 y 126 bis.

21 AIFS, expediente 250, cfr. AIFS, expediente 323.

Cincuenta años

Hispania Sacra 49 (1997) 


\section{FALTA DE FONDOS Y COMUNICACIÓN DEFECTUOSA DENTRO DEL CSIC}

La actividad del IFS se completaba con la edición de las revistas Estudios Bíblicos y Revista Española de Teología. El presupuesto que el CSIC asignaba al IFS resultaba insuficiente para pagar estas ediciones, de modo que, en 1944, estaba prácticamente agotado antes de fines de abril: sobre un total de 161.000 pesetas, quedaban disponibles $2.063,39$, por haberlo gastado en pagos atrasados de nóminas y papel. Eijo tuvo que escribir al Secretario General del CSIC, José María Albareda, pidiéndole un "milagro":

\footnotetext{
"Creíamos (interpretando yo con inefable optimismo la alentadora frase de V.: publiquen, publiquen, que por dinero no ha de quedar) que el exceso de gastos del año pasado saldría de cualquier parte menos del presupuesto del presente.

Y ahora, ¿qué hacemos con nuestras dos Revistas? ¿Será posible que las hayamos de tener que suspender? Por amor de Dios, haga V. un milagro si es menester, pero no deje de echamos una mano piadosa. Pasara, que no pudiésemos publicar ningún libro este año (que ya sería grande lástima), pero ¡Suprimir las Revistas no lo consienta V.?”
}

Como gran logro (casi lindando con la economía del mercado), el secretario general del CSIC respondía que "un tanto por ciento elevado del importe de suscripciones y venta de libros pasaría al Instituto." Eijo, que no ponía la esperanza de la Teología en la ley de la oferta y la demanda, volvía a insistir y pedía como imprescindibles 116.773 pts. ${ }^{22}$.

A pesar de contar con el consentimiento del Secretario General del CSIC, los problemas del IFS no iban a terminar tan pronto, pues Rafael Balbín, vicesecretario del CSIC, interpretaría exactamente al revés la petición de Eijo, a saber: que el crédito extraordinario concedido al IFS no podía emplearse más que para publicar libros, y no las revistas. Los términos en que reacciona Eijo escribiendo a Blázquez, a pesar de su mesura, dejan bien claro que para él quedaba en entredicho aquella preeminencia que, como se aseguraba desde sus comienzos, pretendía dar el CSIC a la Teología:

"Hable V. con Albareda ya que yo he salido para Alicante, y le he encargado a V. Que le ruego se haga cargo de que no podemos trabajar en el Instituto no sólo sin recursos económicos, sino peor aún, con enorme deuda. Ya este año hemos podido hacer poquísimo, por la deuda del año pasado; si en 1945 va a ocurrir lo mismo, nos dominaría el desaliento. Para que viva el Instituto en tan lamentables condiciones yo preferiría que fuese otro el Presidente. Convendría que tuviesen presente la insignificancia de lo que

22 Carta a Albareda, el 22 de mayo de 1944, en AIFS, expediente 250. Añadía que "si se publicase este año la Soteriología Mariana del P. Bover, cosa que estima convenientísima este Instituto, habría que añadir unas 15.000 pesetas". Ibid. 
gasta el Instituto de Teología, comparado con lo que gastan casi todos los demás. E1 vernos asî es cosa que deprime y desanima. Es amargo que tantas veces me hayan asegurado que con el presupuesto extraordinario se saldaría la deuda y resultar ahora lo contrario." 23

En 1945, el IFS tiene una deuda de 63.000 pesetas, y el CSIC le asigna un presupuesto -"crédito"- de 174.450 pesetas, desglosado en 42.200 pts para personal en nómina, 70.250 para revistas y 62.000 para otros gastos. El 11 de enero de 1945 Blázquez envía un proyecto de presupuesto que prevé la edición de cuatro libros y eleva el crédito a 201.373 pesetas. El IFS seguía en una "encrucijada" cuyos términos expresaba así su secretario, para el caso de que el CSIC no se hiciera cargo de la deuda:

\begin{abstract}
"O no pagamos la deuda, que sería cosa por demás bochornosa y prácticamente irrealizable, y que nos desacreditaría para siempre, o si ja pagamos, como es de justicia, no podremos comprar ni tan siquiera sellos para la correspondencia, es decir, tendríamos que paralizar la vida del Instituto, y publicar las revistas pagando el personal de su propio peculio los gastos de relación del Instituto con otras entidades y con sus propios colaboradores."24
\end{abstract}

La documentación conservada en el archivo del IFS permite suponer que en el rechazo a la agregación de nuevos centros llevaba buena parte del peso la carencia de medios económicos. Sin embargo, esta documentación es incompleta y no permite detallar cómo se superó esta crisis, aunque desde luego puede asegurarse que, en todo caso, fue a cargo de los presupuestos del CSIC. A pesar de la afirmación pesimista antes citada - es decir, descontando el problema económico - el balance que el director del IFS hace de esta época es positivo:

"Resultan las dos Semanas altamente interesantes, y dan la desmentida a los que dicen que si se celebrasen cada dos años podrían acudir más trabajos."2s

Eijo resume en esta frase su concepción sobre el ideal de la investigación teológica en España, tal como lo ve en ese momento. Para él, los clérigos españoles se sienten inseguros para poner por escrito sus trabajos de investiga-

23 AIFS, expediente 323. Que la crisis era económica y no de iniciativas parece indicarlo el que, precisamente en mayo de 1944, viera la luz una revista editada por la A.F.E.B.E., Cultura Bíblica. Cfr. BOOMA, 1944, p. 335-336.

24 AIFS, expediente 323, cfr. expedientes 325 y 343.

25 AIFS, expediente 324, cfr. expediente 343 .

Cincuenta años

Hispania Sacra 49 (1997) 
ción. El obispo de Madrid afirma que esta inseguridad carece de fundamento, manifiesta su confianza en la capacidad científica de los sacerdotes españoles, y ve en la historia española una rica fuente de trabajos. Se entiende, pues, que viera en las Semanas un instrumento catalizador para animar a que un mayor número de eclesiásticos se introdujera en el mundo de las publicaciones científicas. ${ }^{26}$

Si la comunicación entre el CSIC y el IFS no era perfecta, dentro del Instituto -en concreto, entre el director y el secretario - se articulaba de forma satisfactoria la organización de las Semanas de Estudios Superiores Eclesiásticos: principalmente durante el mes de agosto, cuando Blázquez enviaba a Eijo -que pasaba el verano en Vigo- los esquemas de los trabajos presentados, y éste proponía la distribución de conferenciantes. ${ }^{27}$

Eijo no dejaba de hacer valer ante el CSIC lo que consideraba un éxito de convocatoria:

\footnotetext{
"Ya son 16 temas fibres, es decir, 16 monografías producidas en un año. ¿Cuándo se había visto otro tanto en España? Ello se debe a nuestras Semanas, y conviene que lo sepan en Investigaciones."28
}

Mientras los dos personajes claves permanecen durante todo el período de nuestro estudio, en 1946 se renueva el personal del IFS, bien por gracia, al ser promovido Máximo Yurramendi al episcopado y dejar libre la jefatura de la sección de teología, de la que se hace cargo otro catedrático del seminario de Madrid, Ramiro López Gallego; bien por desgracia, al fallecer Daniel García Hughes, ocupando el puesto de vicedirector Jesús Enciso Viana, hasta entonces jefe de la sección bíblica.

Ya en 1947, poco después de trasladarse el IFS a su sede definitiva, Eijo forzaba los presupuestos del CSIC y conseguía un aumento de 20.000 pesetas para edición de libros. ${ }^{29}$

\footnotetext{
${ }^{26}$ Cfr. L. EIJO, "A guisa de prólogo", páginas 7-14 de J. L. LAZCANo Escolá:: Potestad del Papa en la disolución del matrimonio de infieles, Madrid, [VI] 1945, Imprenta de Rivadeneyra, 257 páginas.

27 Cfr. las ocho cartas que Blázquez escribe a Eijo en agosto de 1946, y cómo éste le agradece "su diligencia y solicitud y el trabajo que todo ello le ha costado. Dios se lo pague. Ya comprenderá la tranquilidad que todo ello me da y cuán reconocido le quedo" (17 de agosto de 1946). El prelado aclaraba que su consejo no era "cosa que se deba hacer. Estúdielo y dígame o haga lo que mejor crea" (22 de agosto de 1946). AIFS, expediente 365.

28 Tanto en 1946 como en 1947, las Semanas recibieron un crédito de 15.000 pesets por parte del CSIC. AIFS, expediente 365 , cfr. expedientes 379 y 364 .

29 AIFS, expediente 1095 , cfr. expedientes 418 y 440 bis.
} 


\section{SENTANDO CÁTEDRA}

El papel de Eijo en las Semanas, en lo que tiene de doctrinal, se manifiesta particularmente en el saludo que dirigía a los asistentes -"semanistas"- al comienzo, cada año. Con el tiempo, irá delegando muchas de sus funciones: deja de asistir a las sesiones y encarga a otras personas - por ejemplo su obispo auxiliar, Casimiro Morcillo- que lean el saludo. Pero procura siempre escribirlo y, si en muchos casos es propiamente un mero saludo, en ocasiones excepcionales constituye una auténtica intervención magisterial.

En el saludo de 1947, Eijo parece afirmar que la asamblea que consituyen las Semanas es una escuela, y que lo decidido en ella sienta cátedra, al criticar una teoría que "fue rechazada en la Semana del año pasado, y como definitivamente resuelta la cuestión queda para siempre extramuros de nuestras Semanas Teologicas". 30

En el saludo de 1948, Eijo se refiere a las disputas teológicas que estaban teniendo lugar en Francia - da la impresión de que la frontera francesa con España, cerrada totalmente entre marzo de 1946 y febrero de 1948, había sido impermeable incluso a las publicaciones - acerca de lo que se daría en llamar Nouvelle Théologie.

El origen inmediato de esta polémica estaba en el dominico Marie-Dominique Chenu (1895-1990), que en 1920 empez6 a enseñar en las aulas de la escuela de Le Saulchoir, profundizando en las relaciones entre "el Evangelio y el tiempo". Chenu, interpretando "los signos de los tiempos", en opinión de Illanes, dejaría ver en el trasfondo de algunas de sus afirmaciones "una comprensión de la historia que deriva, al menos en parte, de planteamientos hegelianomarxistas no suficientemente criticados."

En 1946, Chenu fue nombrado profesor en la Sorbona (hasta 1953), a pesar de que cuatro años antes había tenido que abandonar su cargo de regente en Le Saulchoir, al incluirse una de sus obras en el índice de libros prohibidos. La polémica se avivó con la propuesta "de un reentronque con la teología patrística por parte de los jesuitas de Lyon-Fourvière, "considerada por algunos críticos como el anuncio de 'una nueva teología', a la que afectaban el relativismo, o incluso un velado agnosticismo". En 1948, el debate habría disminuido en intensidad, "aunque la situación continuó tensa, particularmente en Francia, a la que la discusión había quedado circunscrita."31

\footnotetext{
30 AIFS, expediente 440 bis. La memoria del Instituto para el VIII pleno del CSIC (1947) en AIFS, expediente 418.

31 J.L. ILLANES, Historia de la Teologia (en colaboración con J.I. SARANYANA), Madrid, 1995, BAC, 404 páginas, p. 362. Las referencias a Chenu en p. 329-330.
}

Cincuenta años

Hispania Sacra 49 (1997) 
Así pues, la intervención de Eijo parecía llegar cuando la polémica decaía. Ese año se celebraba un sínodo en la díccesis de Madrid, y el obispo no tuvo tiempo ni para dar a Blázquez una opinión sobre quién debía moderar las sesiones de las Semanas. En cambio, escribió el que sería su más extenso saludo a los semanistas, y también el de más violenta combatividad antimodernista, criticando que "Revistas francesas católicas, en artículos firmados por creyentes y militantes y sacerdotes, ya seculares ya regulares, y no de paso, a la ligera e inadvertidamente sino con reiteración y tenacidad y el ardimiento de las polémicas, pintaban con sombríos colores tan desolador y desastrado cuadro de nuestra Santa Madre la Iglesia Católica, que se llenaba de pavor el ánimo".

Eijo se refiere a España como "alcázar de la ortodoxia", pero la reconoce sensible a la influencia francesa, pues, "en sus tiempos de decadencia y postración dio en hacerse discípula de otros pueblos en los que el instinto humano prevalecía sobre el espíritu y criterio de lo divino, y el interés terrenal de la Patria inspiraba hasta la alianza con el turco con tal de aislar, acorralar y atemorizar a una Nación que prodigaba el oro de sus arcas y la sangre de sus hijos en defender la fe católica en el mundo":

"El mal es ciertamente grave. No me refiero aquí a la labor demoledora y asfixiante de la escuela única, oficial y laica que en el transcurso de dos o tres generaciones ha dejado reducido el número de los católicos franceses, los de ideas y prácticas netamente catolicos, a una quinta parte de los habitantes del país; aludo solamente a una fermentación ponzoñosa que trabaja el seno de esa minoría católica.

Ante la creciente descristianización de la gran masa popular, especialmente la obrera, en vista del abismo cada vez más hondo y ancho que media entre el cristianismo y las neopaganizadas sociedades modemas, varios grupos, solamente unidos por común ribete o marchamo de fervor apostólico y de ansias reformistas, pero fragmentados y aun opuestos por ideas y programas, acusan y denuestan a la comunidad cristiana, osando contra la jerarquía sagrada, clamando contra un rutinarismo amortecedor, un desplazamiento de la realidad contemporánea, y trazan de la Iglesia una imagen como de una energía degradada incapaz ya de salvar al mundo, y a la cual no le queda sino este dilema: o reformarse intima y radicalmente o perecer."

[...] Ven a cuatro quintos de la nación ajenos a la Iglesia y quieren que ésta se transforme para que sea aceptada. Se preconizan adaptaciones, que podrian llegar a claudicaciones en lo fundamental; se habla contra las fómulas dogmáticas con las resbaladizas y capciosas frases del Modernismo anatematizado por Pio X; se alardea, frente a la autoridad, del gallarda independencia bordeando la rebeldía; se dice que hay que despertar a latigazos a los católicos durmientes, pero ¡cuántas veces resulta flagelada la misma Madre Iglesia!; reprochan a los católicos de aletargados inactuantes, pero recriminan de ello al clero y a los Obispos, que los embelefian con novenas y cultos estériles. Saben a protestantismo naciente las osadas frases con que presentan en contradicción con los Apóstoles la Jerarquía eclesiástica de hoy. Y así, resbalando y descendiendo en tan peligroso plano de deslizamiento, ya no se contentan con reprochar a los catolicos, al clero y a la persona del Obispo, inculpan a la misma Iglesia. Menos mal si alguno atenúa di- 
ciendo: 'al menos esta Iglesia de Francia'; pero así y todo, es triste que mostrando celo religioso se pisotee el $4^{\circ}$ Mandamiento de la Ley de Dios."

Eijo explica que, para "inmunizar a nuestras juventudes" contra "el desaliento en el apostolado" que podría provocar esta polémica, había escogido para la reflexiớn de las Semanas de 1948 una frase de Pro XII - "ese don de Dios a la humanidad en estos días"-: "La Iglesia es siempre joven; tiene la fuerza de Dios y no puede envejecer."

Las Semanas no eran una asamblea doctrinalmente indefinida, como ya hemos visto, y además sus puntos de partida y conclusiones depenđían de una referencia concreta: la doctrina del Papa. Pero, según Eijo, tampoco trataba de repetir frases hechas, sino de fomentar una auténtica reflexión "para que pensemos rectamente y para que en nuestros métodos de apostolado cambiemos tanto como hace falta cambiar". El propio Eijo aporta en este saludo su interpretación personal sobre el fenómeno que ha provocado el debate en el país vecino:

"Es verdad; allí y mucho más todavía en España, esas seducidas masas obreras no rechazan a Cristo ni su doctrina; sienten aversión, y aun a veces tan solo desconfianza, hacia la Iglesia, y mejor diría hacia sus representantes, casi siempre envenenados por interesadas campañas partidistas que no hemos sabido contrarrestar, a veces también por equivocaciones de conducta y por malas obras que ¿quién lo duda? Dios nos tendrá en cuenta y cargará en nuestro Debe.

Pero sean cuantos sean y en el grado que lo sean los apóstatas, no son ramas que se han secado y han muerto por falta de savia vital en el árbol, que es la Iglesia; han sido desgajadas de él, que sigue siempre rebosante de vida." ${ }^{23}$

\section{LA MODERNIDAD TIENE SUS LÍMITES}

España va saliendo del aislamiento: hasta el giro que supondrán el gobierno de 1957 y el Plan de Estabilización de 1959, el régimen de Franco pasa por una etapa de éxito político cuyo exponente más representativo es el año 1953, con la firma de los acuerdos con los Estados Unidos y el concordato con la Santa Sede. En el IFS no se producen cambios trascendentales en esta década y media a la que ahora nos referiremos - hasta la muerte de Eijo en 1963-, aunque percibamos al menos a partir de 1955 un balance en parte contradicto-

${ }^{32}$ El texto completo de este saludo en AIFS, expediente 591. La memoria (30 de diciembre de 1948) presentada por el IFS al IX pleno del CSIC, en AIFS, expediente 594.

Cincuenta años

Hispania Sacra 49 (1997) 
rio, por lo que se refiere a la valoración de las Semanas, entre Eijo y Blázquez. Importa reseñar el cambio de ministro de Educación en 1952 - Ruiz Jiménez sustituye a Ibáñez Martín, con quien Eijo mantenía excelentes relaciones-, aunque este cambio no tuviera trascendencia en la vida del IFS.

Las Semanas de 1949 contaron con unos trescientos asistentes, entre los que no se contó Eijo, quien encargó ese año el saludo a los semanistas al ya mencionado Jesús Enciso Viana. Quizá el hecho más relevante fue la propuesta que hizo Blázquez a Eijo de crear una sección de Teología española - en una carta que el secretario escribe al obispo el 19 de agosto-, pues permite ver cómo el ya más que septuagenario obispo sabía concretar las ideas - la sección comenzaría a trabajar en diciembre de ese año- y quizá también una visión más moderna que la de su principal colaborador:

"Me encanta la idea de la Sección de Historia de la Teología española. Iniciar, coordinar, intensificar los trabajos para teger [sic] esa Historia sería tarea muy propia de nuestro Instituto, y grandísima gloria el lograrlo.

[...] Sólo la Historia de los Centros (Estudios Generales y Facultades) ya lo sería; cuánto más llegar a las individualidades y a los pormenores doctrinales. Yo comenzaría por los Centros.

Con nuestros actuales recursos sería inútil acometer tamaña empresa; para obtener mayor y proporcionado aumento de dotación convendría que yo llevase al Ejecutivo un programa más pormenorizado y esquema de reparto de trabajo, con método de subvención del personal; eso convencería; simple petición mediante somera enumeración, lo dudo.

Claro que tras lograr ese empeño de teger [sic] tal Historia el Corpus Theologorum Hispanorum sería cosa relativamente fácil. Como el Corpus, si ya lo tuviésemos, facilitaría la Historia.

Lo que no me llena es el título de Teología Española; me gustaría más Teología Católica en España; téblogos españoles está bien; pero Teología Española, no." ${ }^{33}$

Así pues, no hay nacionalismo en los planteamientos del obispo de Madrid, y sí vinculación manifiesta con el magisterio católico. Los temas "imperiales" dan paso a otros de mayor actualidad, como evidencian los temas de las Semanas de 1950: la Bíblica tuvo como tema central la restauración del Estado de Israel —que en 1946 había sido rechazada como carente de interés - - y el centro de atención era el profesor converso Eugenio Zolli, que presentaba un tema

33 AIFS, expediente 820. Aunque les fue pedida (AIFS, expediente 823) y aparece citada en la de 1950, no parece conservarse memoria de la labor del Instituto en 1949. Hay, sin embargo, un resumen de las actividades desarrolladas entre 1940 y 1949 , por el que sabernos que ese año se incorporaron a la nómina del Instituto Andrés Avelino Esteban Romero como jefe de la sección bibliográfica y María del Carmen Reguera del Mazo como administrativa. AIFS, expediente 1095.

Cincuenta ar̃os Hispania Sacra 49 (1997) 
libre. Eijo explicaba así el cambio de actitud respecto a la cuestión judía, "de grave importancia y además utilísima por su actualidad":

"De grave importancia, porque atañe al amado Israel, el pueblo escogido por Dios en la descendencia de Abrahán por Isaac y Jacob, que entre todos los pueblos de la tierra tiene la gloria de contar con Jesús, María y José. Por él llegan hasta nosotros las bendiciones de Dios; en él estamos ingertados [sic] para dar frutos de vida etema.

[...] Mas he dicho que era también materia utilisima por su actualidad. En efecto, el Sionismo ha logrado ya lo que desde hace veinte siglos anhela; logrolo, pero mísera y efimeramente en el siglo IV, de manos de Juliano el Apóstata; no sabemos el porvenir de ese logro de hoy, es decir, si se afianzará y perdurará; pero debemos estudiar ese hecho, que hoy es ya una realidad histórica, por lo que atañe a nuestra fe cristiana." ${ }^{34}$

Y si el estudio de un tema de actualidad era síntoma de modernidad en la Semana de Estudios Bíblicos —que, además, se anteponía por primera vez a la de Teología-, en ésta se volvían a poner de relieve los límites de la "Teología Nueva", a la que Eijo da ya este nombre en su saludo a los semanistas. El obispo defendía la armonía entre fe y razón, que entendía atacada por el fenómeno que criticaba, y por eso veía una crisis moral donde otros veían sólo necesidad de cambio teológico: rosa.

"El irracionalismo atribuido a nuestra fe la condena a muerte y por cierto poco hon-

El asunto es de suma importancia. Nuestra fe está sobre nuestra razón, pero no contra nuestra razón, ni siquiera es ajena a ella, no puede nuestra fe prescindir de nuestra razón. Hay mutuas interferencias entre ambas; tienen zonas comunes, hay verdades plenamente asequibles a nuestra inteligencia que han sido reveladas por Dios y que para ingentes muchedumbres no pasarán nunca de artículos de fe."

Eijo aprecia el valor de lo nuevo - "muchos conceptos y muchísimas expresiones que hoy tenemos por consagradas fueron un día novedad en la Iglesia"-, aunque le da un matiz principal ciertamente negativo y, en último término, se remite al criterio magisterial, imprescindible en una ciencia que presupone la fe:

"Así como no hubieran nacido nuevas formulas dogmáticas, que clara y luminosamente contornan las verdades de fe, si no las hubiesen precedido nuevas heregías [sic], así acaso algunos conceptos y algunas expresiones del Ángel mismo de las Escuelas [Tomás de Aquino] que pueden desarrollarse, ajustarse, precisarse más por el contraste con formas doctrinales equivocadas, algunas de las cuales no son nuevas sino nueva-

34 AIFS, expediente 1091.

Cincuenta ẵos

Hispania Sacra 49 (1997) 
mente puestas en circulación, y también con expresiones modernas de sabor y apariencias científicas y hasta brillantes, pero ambiguas; tal vez no las rechazan los acatolicos, porque van bien con sus doctrinas; si por esto las adoptáramos, no los habríamos convertido a la fe cristiana, habríamos sembrado babélicas confusiones.

¿Cómo debe discriminarse el oro de ley de la verdad del oro falso del error o del oropel de la mera brillantez literaria? Estudiando, investigando, comparando a fondo lo nuevo con lo tradicional, y sobre todo mediante el fiel contraste del Magisterio eclesiástico."

Tras este preámbulo metodológico, viene el mencionado juicio sobre la "Teología Nueva", negando su premisa mayor:

"No creo que se pueda poner en duda la buena voluntad y el celo religioso, discreto y acertado o desacertado e indiscreto, que inspira a muchos de los innovadores; pero sî creo que debe ponerse en tela de juicio si la paganización cada día mayor en algunos pueblos cristianos obedece a repugnancia - como se asegura- hacia fórmulas, expresiones doctrinales, dogmas, en último término a las creencias, a la fe, siendo como es tan necesario, tan natural, tan usual el creer, el tener fe, así en lo humano como en lo divino; o si más bien esa paganización obedece a que molestan los diez mandamientos, sobre todo los cuatro últimos, y a querer la gente sacudir y borrar la Ley de Cristo, que no tienen por yugo suave y carga ligera. La sensualidad, diosa que hoy lo invade todo, enerva, bajo su imperio fenecen las energías; y sin energía viril toda carga, todo yugo, aunque el amor los haga llevaderos, resultan insoportables." 35

La crítica de Eijo estaba en la línea que iba a tomar la encíclica Humani generis de Pío XII... Lo que no significa en la línea de la teología que iba a tener más éxito:

"La Humani generis es, sin duda, muy sensible frente a cuanto puede conducir al relativismo, y subraya, por tanto, de forma decidida, la conciencia que la Iglesia tiene sobre la verdad de su fe y el valor de su magisterio. Su tono es neto y, en ocasiones, fuerte. Aunque fue seguida - por parte, sobre todo, de los superiores de las ordenes dominicana y jesuita-, de medidas disciplinares bastante duras - apartamiento temporal de la docencia de los autores que, de una forma u otra, estaban más directamente relacionados con los planteamientos objeto de crítica--., la reflexión teológica continuó creciendo y en la década de 1950 se publicaron algunas de las obras más representativas de la teología de mediados de siglo: Jalons pour une théologie du laïcat, de Congar; Méditation sur l'Église, de De Lubac; Essai sur le mystère de l'histoire, de Daniélou; los primeros volúmenes de los Schriften zur Theologie, de Rahner..." 36

35 Saludo a los semanistas manuscrito por Eijo, septiembre de 1950, AIFS, expediente 1091.

36 J.L. ILLANES, Historia de la Teologia, p. 363. Yves-Marie Congar, nacido en 1904, pertenecí́ a la escuela de Le Saulchoir y fue perito en el Concilio Vaticano II. Juan Pablo II lo creó cardenal en 1994. Los dos jesuitas citados son los principales autores de la comunidad de Lyon-Fourvière: Henri 
Aún considerando que una visión excesivamente crítica no permitiera a Eijo ver los aspectos positivos de la "Nueva Teología", no se puede negar que percibió tempranamente la trascendencia que podía tener. Así se explica que centrara los estudios de las Semanas de Teología precisamente en la encíclica Humani generis.

Eijo de nuevo elaboraría el saludo para las Semanas de 1952, con tal interés que, ese año, se adelantó en la correspondencia al secretario del IFS, pidiéndole los programas de las Semanas de 1948 y 1949, "porque ya entonces empezamos a meternos en la harina que requirió la Humani generis". Se trataba ahora, de referirse al movimiento ecuménico, frente al que Eijo aconseja una precaución activa. El obispo de Madrid, de nuevo, no encontraba nada doctrinalmente valioso en lo que calificaba de irenismo que pretende "reconciliar dogmas opuestos":

"Pero lo dificil es encontrar el medio de unirse. Rozar y desmochar las creencias diferenciales para conseguir un credo común es ilusorio. Me recuerda la simplista y vana tentativa de Justiniano $\mathrm{II}^{\circ}$, cuando, viva todavía la lucha nestoriana y en pleno combate, por otro lado, con el monofisismo, buscando la paz y la unidad dictó su decreto de 565 ordenando el olvido de todas esas disputas y exhortando a todos los cristianos a que se contentasen con alabar al Salvador, sin empeñarse en dar de Él explicaciones claras y definidas.

Nunca será ése el camino.

Ni podrá haber unidad religiosa sin autoridad Magistral, ni la autoridad Magistral podrá carecer de cabeza suprema ni podrá ejercer esa autoridad el pueblo piadoso como quieren los modernos teólogos del Oriente separado o como enseña la doctrina protestante con su inspiración privada. Cristo mandó a sus Apóstoles a ensefiar a todos no a que todos los enserfasen.

[...] La mentalidad predominante en el actual Movimiento Ecumenista se cifra en que ninguna de las actuales confesiones cristianas es de por sí la Iglesia, Una, Santa, esposa de Cristo y cuerpo suyo místico; y que por tanto la unidad no se ha de lograr por conversiones individuales a alguna de ellas; esa Iglesia de Cristo hay que realizarla por la unión de las diversas hoy existentes, arrepentidas y enmendadas de su pecado de división.

[...] Por amor a Cristo amemos a nuestros hermanos disidentes; con caridad sobrenatural oremos por ellos, como nos inculca nuestra Madre la Iglesia; deseemos ardientemente su retorno a la casa paterna; evitemos cuanto pueda alejarlos más; pero nunca

de Lubac (1896-1991), también perito en el Vaticano II y cardenal desde 1983, que había publicado en 1938 Catholicisme y en 1946 Surnaturel, donde criticaba el "aristotelismo rígido" de Cayetano y Suárez; y su discípulo Jean Daniélou (1905-1974), impulsor de la colección Sources chrétiennes, perito conciliar y cardenal (1969). Cfr. J.L. ILLANES, Historia de la Teologia, p. 330-334.

Los 16 volúmenes de escritos teológicos de Karl Rahner (1904-1984) no comenzaron a publicarse hasta 1954, y por lo tanto no podía Eijo hacer referencia a ellos, aunque sin duda el "giro antropológico" de este autor tiene que ver con la cuestión tratada. Cfr. ibidem, p. 339-343.

Cincuenta ẵos

Hispania Sacra 49 (1997) 
nos dejemos llevar de falso irenismo que en vez de atraerlos a la Verdad nos aparte de ella a nosotros." 37

Ningún asomo, pues, de aceptar nuevos planteamientos doctrinales, aunque sí se acertara en el meollo de las cuestiones al menos en la elección de los temas, como es manifiesto en el caso de la Semana de Teología de 1953, que llevaba por título Teología del laicado. El planteamiento que hacía Blázquez, en una entrevista de prensa, no era desde luego innovador:

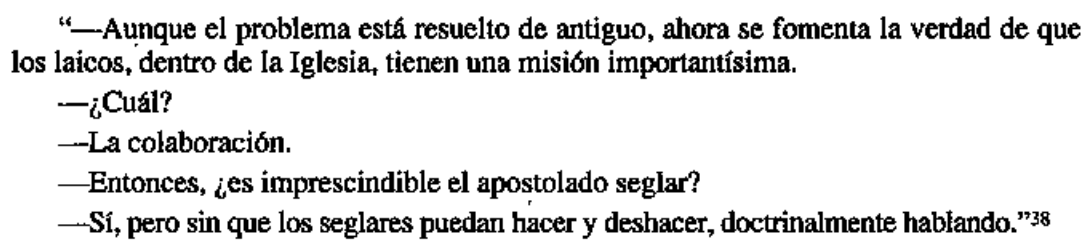

Aunque no hubiera grandes novedades doctrinales, el IFS avanzaba en otros campos, y en 1953 se ponía en marcha algo que en otros tiempos había constituido un sueño para Eijo: "una escuela de lenguas semíticas". ${ }^{39}$

En 1954, la novedad consistió en el traslado del lugar de celebración de las Semanas, que permutaron los calores madrilenos por los aires frescos de Santiago de Compostela. Se trataba de aprovechar la coincidencia del Año Mariano en la Iglesia universal con el Año Santo Compostelano, para mayor gloria de un concordato al que Eijo calificaba - no se conserva el texto de su saludo, pero sí una reseña- de "punto de partida para centrar las distintas reacciones en torno a la naturaleza jurídica de la Iglesia y sus Derechos. Lamentó la influencia que en los medios católicos españoles está teniendo ciertas tendencias extranjeras en torno a las relaciones de la Iglesia con el Estado."40

$Y$, por lo que se refiere a las actividades al margen de las Semanas, también el esfuerzo de Eijo apuntaba a la simple reedición de la doctrina tradicional: consideraba que el "más cumplido homenaje del Consejo [CSIC] a la Inmaculada Virgen" en el Año Mariano sería la publicación de las obras Sacra Deiparae, del mercedario Saavedra - "lo mejor publicado sobre la Inmaculada en

\footnotetext{
37 AIFS, expediente 1531.

38 Informaciones, 21 de septiembre de 1953. AIFS, expediente 1761.

39 AIFS, expediente 1761. Cfr., expediente 1783.

40 AIFS, expediente 1973.
} 
nuestro siglo de oro"-, y De Virginitate Perpetua, "una de las más grandes glorias de la Iglesia en España". ${ }^{41}$

\section{¿"ALTA INVESTIGACIÓN CIENTÍFICA" O “REPETIR LO SIEMPRE DICHO”?}

Con lo hasta ahora visto -es decir, limitándonos a una somera descripción de las publicaciones y los temas tratados en las Semanas, a las exposiciones de Eijo, y sin examinar la calidad del trabajo de los "semanistas"-, podemos quizá anotar algunos evidentes aspectos positivos y negativos del IFS: entre los primeros, una indudable activación de los trabajos teológicos y bíblicos a nivel nacional. Entre los segundos, el hecho de que buena parte del trabajo organizativo - aunque no se manifestara afán centralista- recayera sobre una (Blázquez) o dos personas (sumando a Eijo).

En la preparación de las Semanas de 1955, Eijo no pudo intervenir en absoluto. Con motivo de la retirada de uno de los ponentes, Blázquez se preguntaba si no habría que evitar la rigidez en el planteamiento temático ${ }^{42}$.

Las angustias que pasaba el secretario del IFS para conseguir que se le enviaran a tiempo trabajos para las Semanas contrastan con el optimismo con que Eijo pintaba el panorama de las ciencias eclesiásticas en España:

\footnotetext{
"Nuestras Universidades y Facultades eclesiásticas sostienen muy aita la bandera de la ciencia sagrada, según lo han reconocido eminentes pensadores europeos. Cuentan con modestas subvenciones y escasos recursos; y, a pesar de esto, sus bibliotecas aumentan, reciben centenares de revistas científicas; una de ellas, por poner algún ejemplo, 630; otra, 422; publican colecciones de alta investigación científica y revistas muy acreditadas en España y en el extranjero; envían delegados a Congresos Internacionales; por ejemplo, la Asamblea de Universidades Hispánicas, en octubre de 1953; al Congreso Científico de Roma en el mismo mes de 1954, al Congreso Argentino de Psicología, al de Filosofia de Sao Paulo, al de Filosofia de las Ciencias en Zurich."43
}

En 1956 no hubo especial dificultad en la organización de las Semanas -el discurso de apertura fue de Eijo-, aunque coincidieron con una crisis en la AFEBE. A través de la documentación existente, puede verse cómo Eijo, a pesar de haber dado vida a estas iniciativas y de tomarlas con entusiasmo, no

\footnotetext{
41 AIFS, expediente 1984.

42 AIFS, expediente 2151.

43 BOOMA, 1955, p. 165.
}

Cincuenta ax̆os

Hispania Sacra 49 (1997) 
las emprendía con afán acaparador, y se interesaba por que otras personas tomaran parte y decidieran sobre ellas. ${ }^{44}$

Será en 1957 cuando encontremos la primera expresión abierta de una valoración distinta de la de Eijo, por parte del secretario del IFS. Por una parte, el trabajo recae cada vez más sobre Blázquez, ya que el ritmo de trabajo del obispo de Madrid se ha frenado sustancialmente: ese verano tarda casi dos meses en escribir a su secretario personal, Juan Botella, para pedirle documentos relativos a la vida de la diócesis. ${ }^{45}$

Pero no es al exceso de trabajo a lo que hace referencia Blázquez en su primera valoración genérica negativa —al menos en parte- del resultado de las Semanas, cuando escribe al obispo ausente:

\footnotetext{
"Hay muchas caras nuevas en sí y para nosotros. Faltan algunas caras venerables en sí y para nosotros. Claro que la mayoría de estos últimos fueron estrellas fugaces, por desgracia, en nuestras Semanas. [...] Muchos van sólo a mirar, pero algo recordarán con ese motivo. Los trabajos mantienen un tono digno. Quizá se les pueda achacar un desenfoque en el planteamiento, que trae como consecuencia un repetir lo siempre dicho sin avance positivo en la solución del problema. Las discusiones muy animadas y con gran contento de todos. Sin embargo diría que adolecen de falta de preparación próxima en la inmensa mayoría y a ratos dan la sensación de que se está improvisando apoyados únicamente en la dialêctica." 46
}

La escasez de recursos - de personal o propiamente económicos- en el IFS era manifiesta. Ese verano, la publicación de los volúmenes con los trabajos de las Semanas recibió una subvención de 90.000 pesetas anuales por parte del CSIC. Blázquez no daba abasto para publicar las actas: el 27 de diciembre de 1957, Rafael Balbín, vicesecretario del CSIC, le amonestaba por planear la publicación (para 1958) de las actas de la Semana de Teología de 1956 y de un trabajo de la Semana Bíblica de 1955.47

Ya en 1958, veremos a un Eijo octogenario que -aunque aún viviría cinco años más- escribe a su secretario personal, con la mirada puesta en el otro mundo:

\footnotetext{
"Ya reacciono mucho menos a los medicamentos. Lo cual, te digo con sinceridad, me alegra pensando que está más próxima mi suspirada resolución (S. Pablo) y el estar
}

44 AIFS, expediente 2296; cfr. BOOMA, 1957, p. 141

45 Cartas a Juan Ignacio Botella en el archivo de la secretaría de la Archidiócesis de MadridAlcala (ASDMA), estantería izquierda, carpeta XVI.

46 AIFS, expediente 2422.

47 AIFS expediente 2425 . 
con Cristo. Me emociona de amor el pensarlo, y si no lo deseo es porque no quiero querer más que lo que Él quiera." 48

Lo cierto es que, si hemos tenido que buscar intensamente para encontrar diferencias de puntos de vista entre Eijo y Blázquez o algún atisbo de desánimo en la organización de las Semanas, no podemos dar demasiada importancia a estos problemas. El verano de 1958 fue, precisamente, el primero en que el secretario del IFS se tomó un descanso en la organización de las Semanas ${ }^{49}$.

En 1959, Blázquez encuentra de nuevo tiempo para buscar en la Biblioteca Nacional de París materiales útiles para la historia de la teología en España. Los participantes en las Semanas, como siempre, se retrasaban en la presentación de esquemas: "No sé si serán los calores o las tormentas, pero nuestros hombres andan este año más recalcitrantes que nunca", escribía Blázquez a Eijo el 31 de agosto 1959. La Semana Bíblica, sin embargo, según escribía tres dias antes, "queda bastante completa." 50

\section{LA DESPEDIDA DE EJJO}

En 1961, el obispo de Madrid dirige su último saludo a los participantes en las Semanas de Estudios Superiores Eclesiásticos. En esta ocasión -convocado ya el Concilio Vaticano II-, vuelve a referirse al ecumenismo, tema que trató ese mismo año en una carta pastoral dirigida a todos sus diocesanos.

La comparación entre lo que dice a un público más amplio y lo que reserva para los especialistas de las ciencias sagradas puede servir para comprender mejor sus intervenciones en anteriores saludos a los semanistas: en cuanto director del IFS y en cierto modo representante del magisterio eclesiástico en ese ámbito de las ciencias, sus intervenciones son escasas y, en general, tienen el carácter de reacción y aviso frente a posibles errores. De ahí el tono que puede resultar excesivamente crítico, si pensamos que toda su visión del asunto tratado se reduce a lo que expresa su discurso ante los semanistas.

El caso de 1961 nos permite ver que no es así, ya que en su carta pastoral muestra su alegría por una serie de "hechos muy elocuentes que hubiéramos

\footnotetext{
48 Carta el 20 de agosto 1958, en ASDMA, estantería izquierda, carpeta XVI.

49 AIPS, expediente 2545 .

50 El ya citado artículo de J.M. CASCIARO en La Table Ronde $\left(\mathrm{n}^{\circ} 144\right.$, diciembre 1959, p. 117 122: "Les études bibliques et théologiques dans l'Espagne actuelle") da a entender (p. 121-122) que et trabajo de los especialistas bíblicos tenía mayor nivel que el de los télogos. Las demás referencias, en AIFS, expediente 2691 .
}

Cincuenta años

Hispania Sacra 49 (1997) 
juzgado imposibles a principios del presente siglo y que nos demuestran la profunda y maravillosa labor del Espíritu Santo para preparar el camino de la unidad cristiana", instaurando el diálogo "donde antes había un silencio frío y quizás despectivo":

"Una especie de aurora de caridad envuelve en su hz esperanzadora las relaciones entre los cristianos."

En el saludo a los semanistas de $1961-$ a los que se había propuesto como tema de estudio "la Tradición divina"-, por contraste, volvemos a ver las advertencias críticas características de intervenciones anteriores. Eijo se centra en los errores que cometían, en su opinión, algunos ecumenistas:

"Porque si bien por una parte parece quie entre algunos de los más conspicuos teólogos del Protestantismo comienza a remitir y como a desear ponerse en razón aquel odio a la tradicion característico de los primitivos Reformadores, es manifiesto que por otra parte van aumentando algunos autores católicos que ponen en cuarentena ciertos aspectos de la Tradición que unánimemente se tenían antes por indiscutibles. ¿Será de nuevo el irenismo, hijo bastardo de la caridad cristiana, quien debilite la firmeza de la verdadera doctrina? Bien está que con juicioso espíritu crítico se desestime la autoridad de la Tradición mal fundada, y sin más que aducir un par de sentencias de Santos Padres se dé por probada en virtud de la Tradición una tesis teológica. El certero criterio no desvirtúa la Tradición, corrige el mal uso de ella. pero menguar la fuerza de la Tradición en sí misma es desentonar de todo el ambiente cristiano de los primeros siglos y minar la cimentación de nuestras creencias." 52

Y si en su saludo a los semanistas, Eijo podía expresar con mayor claridad que ante sus diocesanos algún punto de vista crítico --desde el punto de vista de la metodología científica, pero también pastoral--, mayor confianza e interés encontramos en la correspondencia preparatoria de las Semanas que mantiene con Joaquín Blázquez. 1962 será el último año en que mantengan esta correspondencia --Eijo murió el 31 de agosto de 1963--, y en ella es primero el secretario del IFS quien recuerda al obispo el objetivo de sus reuniones científicas:

"Lo que nos interesa es que no se descienda ni un milimetro de la altura estrictamente teológica en que nosotros debemos situamos, sin fobias ni filias, sin ismos de ninguna clase, sino buscando serena y científicamente la verdad y sólo la verdad. Creo

51 BOOMA, 1961, p. 4. El 30 de diciembre de 1961, firmará una nueva pastoral sobre el mismo asunto, publicada en BOOMA, 1962, p. 3-16.

\$2 Reseña de prensa conservada en AIFS, expediente 2969. En ausencia de Eijo, su saludo fue leído por el Secretario del Instituto. Para las sernanas de 1960, cfr. AIFS, expediente 2840. 
que éste es el mejor servicio que los téblogos españoles podemos y debemos prestar a la Santa Iglesia especialmente en estos momentos preconciliares." 53

En estas cartas que habían de ser de involuntaria despedida, el secretario del IFS hace un balance positivo - que contrasta, o quizá más bien completa, las notas críticas que vimos en 1955 y 1957 - de sus años de trabajo con Eijo, hablando de la numerosa colaboración ofrecida a las Semanas de 1962:

"Es señal inequívoca de la aceptación que han tenido y siguen manteniendo nuestras Semanas que están ya respectivamente por la $22^{\mathrm{a}}$ y $23^{\mathrm{a}}$ edición. Y parece que fue ayer cuando procurábamos aquel primer tacto de codos que fue nuestra $1^{\mathrm{a}}$ Semana Española de Teología. No cabe duda de que es la obra de nuestro Instituto mediante la cual ha influido más en la investigación teológica y bíblica en España y por eso quizá la obra con la que ha llenado mejor su propio cometido." 54

También en su contestación, el 13 de septiembre, Eijo hace algunas referencias a su trabajo en todos esos años, que resultan un imprevisto testamento:

"Me han dado un verano de no parar ni un momento. Gracias que be podido escribir la Pastoral del Domund, que va tegida [sic] de palabras del Papa más que de mías. No he compuesto el saludo para el día 17[...] Si puedo pergeñar en el coche unas palabras, las leeré y si no diré algo de saludo; de modo que D.m. asistiré a la iniciación. Más de uno de los saludos hace años leídos los escribí durante la ida a Madrid.

Me han mandado este año demasiados papeles de ahí, poco he descansado, pero me he repuesto mucho. Realmente Madrid es ya una dícesis que desborda los poderes de un humano, y más cuando éste es de 85 aîos." 55

Como hemos mencionado, Eijo no pudo participar en la preparación inmediata de las Semanas de 1963, cuya celebración se había previsto en Tarragona. El obispo se encontraba en Vigo desde mediados de julio. Salvador Muñoz Iglesias ha relatado sus últimos días, conforme al testimonio del familiar del obispo, Agustín Díaz Sanz. En ellos vemos que el IFS es la única institución

53 Carta a Eijo 3 de agosto 1962. AIFS, expediente 3111.

s4 Carta 25 de agosto 1962. La anterior, también de Blázquez a Eijo, fechada el día 3. AIFS, expediente 3111. Cfr. escritos críticos con el centralismo del CSIC en AIFS, expediente 3277.

55 AIFS, expediente 3111. Juan Zaragūeta aseguraba en 1964 que Ja dotación de medios del Instituto Francisco Suárez "la debe éste en buena parte al celo de su presidente por procurárselos". Luis MARTÍNEZ KLEISER y Juan ZARAGÜETA y BENGOECHEA, A la memoria del Excmo. y Revmo. Sr. don Leopoldo Eijo y Garay Patriarca Obispo de Madrid Alcalá y Presidente del Instifuto de España Madrid, 1964, Editorial Magisterio Español, 29 paginas, p. 24.

Cincuenta afios

Hispania Sacra 49 (1997) 
de las muchas a que perteneció Eijo $-\mathrm{y}$ aparte de su diócesis- que es mencionada en los últimos instantes de vida del obispo:

"El día 30 [de agosto] llamó a D. Agustín y le dijo que le quedaba poco de vida, y que quería manifestar públicamente su agradecimiento a todos sus colaboradores (sus queridísimos Obispos Auxiliares, los empleados de Curia y Secretaría, sus inolvidables sacerdotes, sin los cuales no habría podido hacer nada, sus amigos del Instituto Suárez del C.S.I.C., etc).

Cuántas veces - añadio- he predicado sobre la escena evangélica del Buen Ladrón. Pero nunca como ahora comprendi lo que San Dimas debió sentir: Hoy estarás conmigo en el Paraíso. El cielo, hijo, no es esa cosa lejana por encima de las nubes. Para mí, hoy es algo que está ahí detrás de esa pared a poco más de un metro. Quiero pedirte un favor. Cuando yo muera, procura encender todas las luces de la casa. Quiero que la luz esté reflejando a los que quedáis aquí lo que yo estaré viviendo en aquellos momentos. La muerte no tiene por qué ser tétrica. Es el paso a la luz." ${ }^{\text {s6 }}$

\section{CONCLUSIÓN}

Al IFS le cabe sin duda el mérito de haber dinamizado los estudios teológico-bíblicos en la posguerra española, como prueba la nutrida asistencia a las Semanas de Estudios Superiores Eclesiásticos entre 1940 y 1962, al margen de la consideración que merezca la calídad de los trabajos presentados a estas Semanas, que, como hemos visto, aprecian muy positivamente tanto Eijo como el secretario del IFS, Joaquín Blázquez (éste con algunos momentos en que parece mostrar cierto desencanto, entre 1955 y 1957).

El IFS y las Semanas tenían un claro punto débil: el reducido número de personas sobre las que recaía su organización, y, como consecuencia, el que no existiera una colaboración estable y orgánica con otros centros de investigación (aparte de la que suponía la participación anual en las Semanas).

Otro punto flaco del IFS era su absoluta dependencia económica respecto del CSIC, que le hizo pasar por momentos muy difíciles —en 1944 Eijo escribía que prefería que fuese otro el director del IFS-, y que en todo caso hipotecaba su futuro ante posibles recortes o crisis en el CSIC.

Por último, y refiriénđonos a la disciplina intelectual dentro del IFS y de las Semanas, en un principio Eijo parece haber tratado de formar una escuela con los participantes en estas reuniones científicas, de modo que lo que se resolviera en ellas formara un cierto cuerpo de doctrina. Sin embargo, esto no pasa de ser una expresión aislada, que nunca tuvo consecuencias prácticas. El único

56 Texto redactado y conservado por Salvador Muñoz Iglesias. 
criterio de validez al que se refirió reiteradamente Eijo, y que suponía auténtico compromiso, era el magisterio de la Iglesia católica. Aún así, no hay constancia de que ningún trabajo fuera excluido porque los organizadores lo consideraran incompatible con la doctrina católica.

Obviamente, cabe argumentar que la base sobre la que se asentaba el IFS estaba expuesta a una posible crisis magisterial dentro de la Iglesia. Es una hipótesis que Eijo no parece haber considerado ni siquiera remotamente. De hecho, él garantizó la conexión con esta fuente de doctrina mientras estuvo al frente del IFS, dando por supuesto que, siendo los responsables del Instituto personajes eclesiásticos, la fidelidad a este magisterio estaba garantizada, y con ella la continuidad del IFS.

Cíncuenta años

Hispania Sacra 49 (1997) 